\title{
Production yields of micro-second isomers populated in ${ }^{252} \mathbf{C f}(\mathrm{SF})$
}

\author{
Laurent Gaudefroy ${ }^{1, *}$ and Adeline Ebran $^{1, * *}$ \\ ${ }^{1}$ CEA, DAM, DIF, F-91297 Arpajon, France
}

\begin{abstract}
A twin Frisch grid ionization chamber loaded with an ultra-thin ${ }^{252} \mathrm{Cf}$ sample is used in order to detect spontaneous fission events. The chamber is surrounded by 8 HPGe detectors allowing to perform $\gamma$-spectroscopic measurements. We are interested in the decay of isomeric states populated in fission fragments. In this contribution we report on preliminary results on production yields of isomeric states with half-lives ranging from few nano- up to few micro-seconds.
\end{abstract}

\section{Introduction}

Spontaneous fission sources offer an interesting possibility in order to study a large number of radioactive species that would otherwise require a particle or ion accelerator to be produced and excited. The large amount of unstable nuclei produced in the spontaneous fission process in general, such as that of ${ }^{252} \mathrm{Cf}$ in particular, however makes it difficult to disentangle the measured radiations. Selective experimental setups should therefore be developed in order to perform unambiguous association between radiations of interest and identified fission fragments.

In the present contribution we are interested in the delayed gamma-ray spectroscopy of fission fragments. Micro-second isomers might reflect profound structural changes in the wave functions of low-lying nuclear states therefore inhibiting transition rates between them. Studying the decay of these isomers can allow to locate where these structural changes occur in the Segre chart. With the help of nuclear models one can hope to pinpoint the origin of these changes in a particular region of the nuclide chart [1].

Short-lived nuclear isomers populated during the fission process can also provide interesting information about the fission process itself. Indeed the prompt particle, neutrons and gamma-rays, emission carry some information on the fragment shape configuration, excitation energy and angular momentum at scission. Naively, one expects a clear link between the spin-distribution in the primary fragments and the population of high-spin discrete states in the fragments produced after neutron-emission. In this works we therefore propose to measure the production yield of micro-second isomers in fission fragments. Comparison of these results with theoretical predictions should allow to constrain model parameters on spindistribution in primary fission fragments.

\footnotetext{
*e-mail: laurent.gaudefroy@cea.fr

**e-mail: adeline.ebran@cea.fr
} 
In the first part of this contribution, we present the experimental setup and the achieved selectivity allowing to perform gamma-spectroscopy of identified fission fragments. Then we present the procedure used in order to extract production yields of the populated short-lived isomeric states. Preliminary results are reported for ${ }^{133} \mathrm{Te}$ taken as an example. Perspectives are finally briefly depicted.

\section{Experimental setup and selectivity}

The experimental setup used in the present work consists of a twin Frisch grid ionization chamber loaded with an ultra-thin ${ }^{252} \mathrm{Cf}$ sample surrounded by $8 \mathrm{HPGe}$ detectors. The principle of the measurement is as follows. Both fragments emerging from a spontaneous fission event are stopped in the chamber and their kinetic energy is measured. For cases where at least one fragment is produced in an excited long-lived (from few ns up to few $\mu \mathrm{s}$ ) state, its gamma decay is measured using the HPGe detectors surrounding the ionization chamber. In this section more details on the just sketched experimental setup are reported as well as examples of the achieved selectivity.

Figure 1 shows the twin Frisch grid ionization chamber used in this work. It consists of two sets of axial chambers sharing a common cathode. All electrodes present an active diameter of $165 \mathrm{~mm}$. The cathode-anode distance is $93 \mathrm{~mm}$. Each anode is separated from the cathode by stainless-steel mesh serving as Frisch grids. The anode-grid distance is $13 \mathrm{~mm}$. The chamber is filled with $\mathrm{P}-10$ gas $\left[\mathrm{Ar}(90 \%)+\mathrm{CH}_{4}(10 \%)\right]$ at $900 \mathrm{mb}$. At this pressure the typical range for fission fragment amounts to 2 to $3 \mathrm{~cm}$ and the fragments are stopped within about $3 \mathrm{~ns}$. The cathode voltage is set to $-2100 \mathrm{~V}$; the Frisch grid is grounded and the anode voltage is $+1700 \mathrm{~V}$.

The ionization chamber is loaded with a $15 \mathrm{kBq}$ ultra-thin ${ }^{252} \mathrm{Cf}$ sample. The sample is supported by a $10 \mu \mathrm{g} / \mathrm{cm}^{2}$ carbon backing. This ultra-thin sample has been prepared using the self-transfer technique [2,3].

The ionization chamber is surrounded by 8 coaxial HPGe detectors the relative efficiency of which varies from $40 \%$ up to $80 \%$. The HPGe detectors are vertically centered on the cathode (i.e. on the ${ }^{252} \mathrm{Cf}$ sample) and oriented in the direction normal to the symmetry

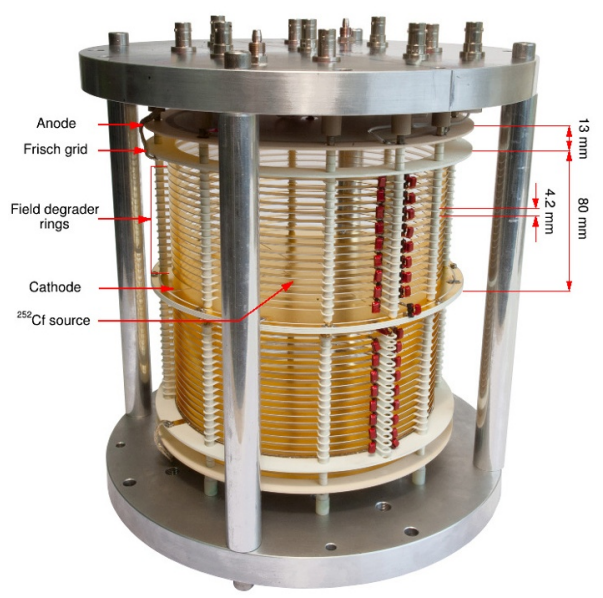

Figure 1. Picture of the twin Frisch grid ionization chamber used in this work. The central cathode support the ultra-thin ${ }^{252} \mathrm{Cf}$ sample. Distances between the electrodes are indicated. 


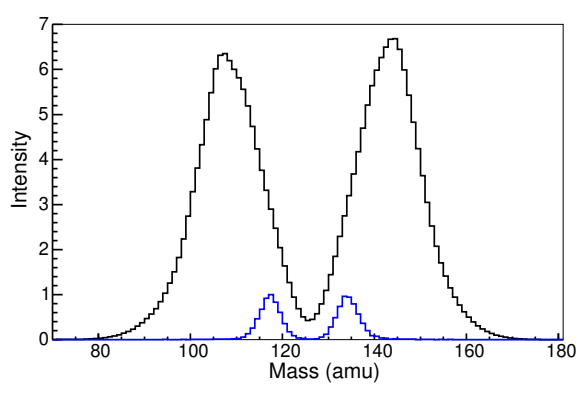

Figure 2. In black, preliminary post-neutron mass-distribution deduced from the measured kinetic energies in the ionization chamber. The blue curve shows the mass distribution gated by one of the gamma rays emitted in the decay of the $6^{+}$ isomer at $1691 \mathrm{keV}$ in ${ }^{134} \mathrm{Te}$. Both curves are arbitrarily and independently scaled. The deduced preliminary mass-resolution amounts to about 5 a.m.u (FWHM).

axis of the chamber. The distance from the symmetry axis of the chamber to the entrance window of the HPGe detectors amounts to $11 \mathrm{~cm}$. The gamma detection efficiency has been determined using standard gamma sources, as well as MCNP [4] simulations accounting for gamma attenuation in the ionization chamber material. The total gamma detection efficiency is maximum at about $150 \mathrm{keV}$ and amounts to $6.2 \%$. It amounts to $1.5 \%$ at $1300 \mathrm{keV}$.

The trigger of the data acquisition system consists of a valid fission event for which both anodes of the ionization chamber detect a signal above the electronic threshold. The later is set just above the ${ }^{252} \mathrm{Cf}$ alpha particles signal. For each valid event, the kinetic energies of both fission fragments are recorded. When present the energy signal of the HPGe detectors are also recorded as well as the time elapsed between the fission event and the detection of the corresponding gamma-ray.

The measured kinetic energies are calibrated in such a way that the mean energy of the light and heavy fragment peaks correspond to the literature values. The present results are still preliminary and corrections for energy-loss in the ${ }^{252} \mathrm{Cf}$ sample; pulse height defect resulting from non-ionizing collisions in the gas and for neutron emission are not yet included. For more details on the complete analysis procedure, the interested reader can refer to Ref. [3]. Figure 2 presents the preliminary post-neutron mass-distribution of the fission fragments deduced from the measured kinetic energies. The blue curve on the figure corresponds to the same mass-distribution gated by the detection of a gamma-ray belonging to the decay of the $6^{+}$isomer in ${ }^{134} \mathrm{Te}$. From the later distribution one deduces a preliminary mass-resolution of about 5 a.m.u. (FWHM) that, combined with the standard energy resolution of the HPGe detectors, provides the selectivity of the present experimental setup. As an example, figure 3 (a)
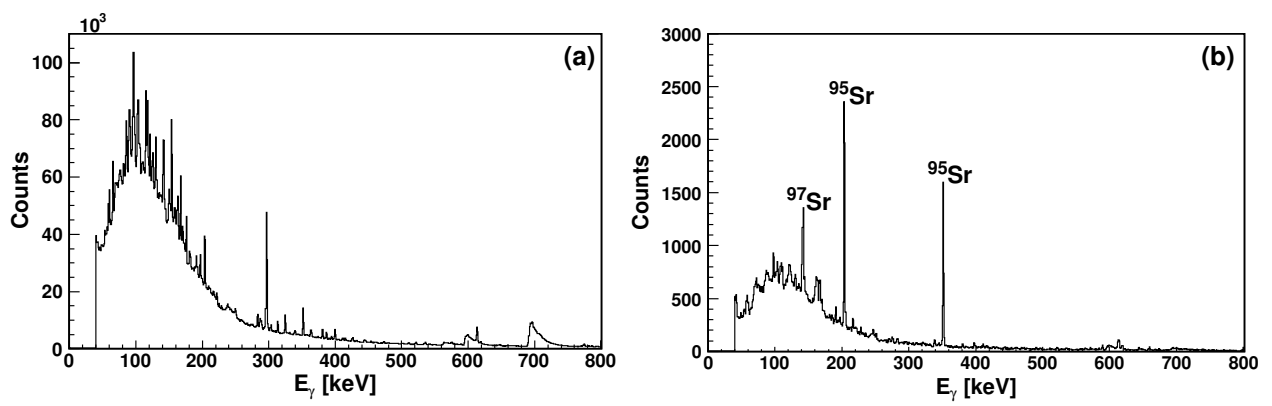

Figure 3. (a) Single delayed gamma spectrum measured in coincidence with a fission event in the ionization chamber. The gamma line is detected from 10 up to $200 \mathrm{~ns}$ after fission. (b) Same as (a) with the additional condition that the mass of the light fission fragment is $\mathrm{A}=95 \pm 0.5$. 
shows the single delayed gamma spectrum measured from $10 \mathrm{~ns}$ up to $200 \mathrm{~ns}$ after a fission event, while figure 3 (b) shows the same spectrum gated by mass $A=95$ in the ionization chamber. In panel (b) one clearly sees the 204 and $352 \mathrm{keV}$ prominent peaks belonging to the decay of the $7 / 2^{+}$isomer in ${ }^{95} \mathrm{Sr}$. These gamma-lines are hardly visible in panel (a) demonstrating the selectivity of the mass gate. Because of the aforementioned 5 a.m.u. mass resolution, one also observes a gamma line belonging to mass $A=97$ in the spectrum of figure 3 (b).

\section{Preliminary results}

In this section we present the procedure used in order to extract isomeric production yields. For each isomer, we construct the mass-distribution of its characteristic gamma-lines. As an example the mass distribution for the $1610 \mathrm{keV}$ isomer in ${ }^{133} \mathrm{Te}$ is reported in figure 4. Each point of the mass distribution is obtained by extracting the number of counts in the characteristic peaks of the mass-gated gamma spectrum. In order to maximize the peak to background ratio, gamma spectra are restricted to events detected up to 3 to 5 times the lifetime of the considered isomer. The resulting experimental mass distribution is then adjusted with a Gaussian function in order to extract the total number of counts in the considered gamma peak. This total number of counts is corrected for the aforementioned time gate applied to the gamma spectrum as well as for the gamma detection efficiency. The analysis is performed for each side of the twin ionization chamber, both sides yielding compatible number of counts within the statistical error bars. The production yield of the isomer is obtained by normalizing the total number of counts in the gamma line of interest to the number of fission events detected in the ionization chamber, considering that each fission event gives rise to two fission fragments.

When applied to the $1610 \mathrm{keV}$ isomer in ${ }^{133} \mathrm{Te}$, this procedure results in a preliminary production yield of $\mathrm{Y}=1.23 \times 10^{-2}(5)$, to be compared to the total production yield of $\mathrm{Y}=1.824 \times 10^{-2}$ for ${ }^{133} \mathrm{Te}$ according to the Wahl evaluation [5]. In the future this value will be compared to theoretical ones obtained, for example, with codes such as FIFRELIN [6] or CGMF [7, 8] aiming at describing the decay of fission fragments. The tentative spin/parity of the $100 \mathrm{~ns}$ isomer discussed above is $19 / 2^{-}$while the ground state spin/parity of the nucleus is $3 / 2^{+}$. From the large spin difference between the isomer and the ground state one expects that their relative feeding in the fission process somewhat reflects the characteristics
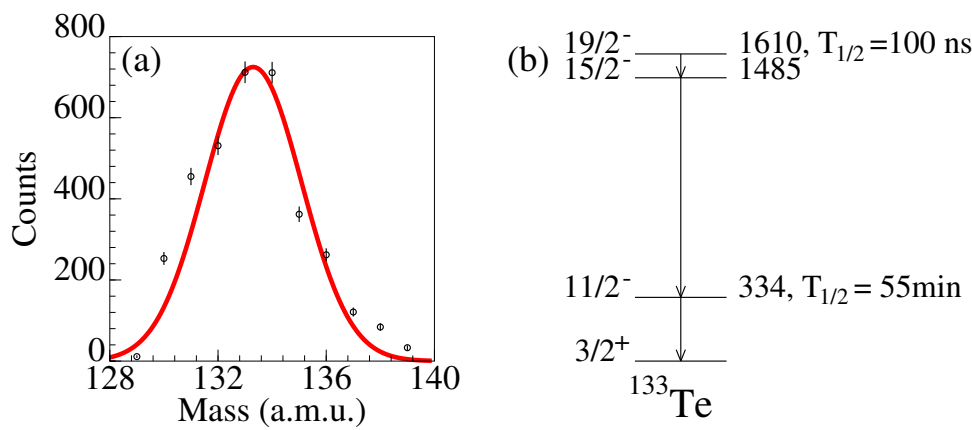

Figure 4. (a) Mass distribution associated with the $1150 \mathrm{keV} \gamma$ transition belonging to the decay cascade of the $100 \mathrm{~ns}$ isomer lying at $1610 \mathrm{keV}$ in ${ }^{133} \mathrm{Te}$. The curve is fitted against the experimental data points. (b) Partial level scheme of ${ }^{133} \mathrm{Te}$ showing the decay path of the $100 \mathrm{~ns}$ isomer at $1610 \mathrm{keV}$. 
of the spin distribution in the primary fission fragments and that present data could constraint related model parameters.

\section{Conclusion}

In this contribution we report on preliminary results on production yields of micro-second isomers populated in ${ }^{252} \mathrm{Cf}(\mathrm{SF})$. The experimental setup consisting of a twin Frisch grid ionization chamber surrounded by 8 HPGe detectors has been presented. The selectivity of the setup for gamma-ray spectroscopic purposes has been demonstrated. About 60 isomeric states have been observed with this setup. The procedure developed in order to extract the production yields of these isomers has been presented for the particular case of ${ }^{133} \mathrm{Te}$.

In the future this procedure will be applied to all the isomers observed in this work. The deduced production yields will be compared to theoretical ones in order to provide constraints on parameters for modeling spin distribution in primary fission fragments.

\section{References}

[1] L. Gaudefroy et al., Phys. Rev. C 97, 064317 (2018)

[2] S. Pauker and N. H. Steiger-shafrir, Nucl. Instrum. Methods A 91, 557 (1971)

[3] L. Gaudefroy et al., Nucl. Instrum. Methods A 855, 133 (2017)

[4] C. J. Werner et al., "MCNP6.2 Release Notes", Los Alamos National Laboratory, report LA-UR-18-20808 (2018)

[5] A. C. Wahl, At. Data Nucl. Data Tables 39, 1 (1988)

[6] O. Litaize, O. Serot and L. Berge, Eur. Phys. J. A 51, 177 (2015)

[7] T. Kawano, P. Talou, M. B. Chadwick and T. Watanabe, J. Nucl. Sci. Technol. 47, 462 (2010)

[8] P. Talou, B. Becker, T. Kawano, M. B. Chadwick and Y. Danon, Phys. Rev. C 83, 064612 (2011) 